\title{
OPERATIONAL AVIONICS INSTRUMENTATION
}

\author{
Tod Reinhart
}

\section{Wright Laboratory, WPAFB OH}

\begin{abstract}
The development of rapid turnaround capabilities to support mission critical Embedded Computer Systems (ECS) is necessary to ensure the most accurate and cost-effective tools are used to enhance the ECS software. Rapid Turnaround (RT) is defined as correcting a system deficiency in a timely manner through some combination of software, firmware, and/or hardware modifications. In order to effectively achieve rapid turnaround of ECS software, the data processed during a mission anomaly must be provided to the radar analyst for mission recreation to ensure appropriate changes are made to the Operational Flight Program (OFP). Anomalous behavior is defined as unexpected avionics system performance. This data requirement has determined the need for an instrumentation system hosted on-board a tactical aircraft to collect and record avionics data. The development of the Data Integration \& Collection Environment (DICE) System by the United States Air Force and TRW is a program designed to address this need.
\end{abstract}

The objective of the DICE effort is to develop a low-cost on-board airborne data collection system utilizing the F-15 APG-63 radar as a proof-of-concept. This collected data will aid system engineers in the analysis of an avionics system's performance and operation by providing a re-creation of the anomaly occurring in an operational environment. As a result, DICE will enhance the rapid reprogramming process of embedded computer systems software.

This paper presents the advantages of an operational avionics instrumentation system, the impacts for deploying such a system field-wide, the potential dual-use of the technology and introduces an operational instrumentation technology development program, DICE.

\subsection{Introduction}

Avionics radar systems containing embedded computer systems (ECS) provide a high level of flexibility in both system configurability and adaptability. However, this increase in flexibility requires a corresponding increase in logistical support. This support includes both hardware and software maintenance. The requirement to provide a rapid turn-around capability to support mission critical ECS in a cost-effective and accurate manner is paramount. In order to achieve the goal of rapid turnaround in ECS software, anomalies occurring during a mission require that specific data processed during the mission be collected and recorded. Operational instrumentation can be used to obtain avionics data from the field which will supplement data acquired during flight test using conventional instrumentation systems.

Once an operational flight program (OFP) has achieved its initial operational capability (IOC), the major reasons for upgrades are 1) to enhance the performance envelop of the baseline software, 2) to improve the electronic combat capability to keep it up-to-date with the operational environment, and 3) to correct latent software errors identified in the field.

\subsection{Background}

The concept of operational instrumentation arose out of the Radar Readiness Technology (RRT) studies conducted by TRW and Hughes Aircraft Company in 1988-89. In 1991, a study of the current test methodologies employed in the isolation, analysis, and resolution of operational 
problem reports (OPRs) was performed to identify areas where state-of-the-art technologies could be adapted to improve the life-cycle support and enhance the Rapid Turnaround process. The Air Force has a goal of 24 hours to implement OFP changes in an emergency cycle. This short period of time allows for only the most elementary of changes utilizing current technologies. It simply takes too long to recreate anomalous behaviors, develop the software algorithms to correct the deficiencies, and test the updated OFP for integrity. In order to better understand the OFP software update process, a flowchart of the process is shown in Figure 1.

The F-15's APG-63 fire control radar system was chosen as the target system for the DICE

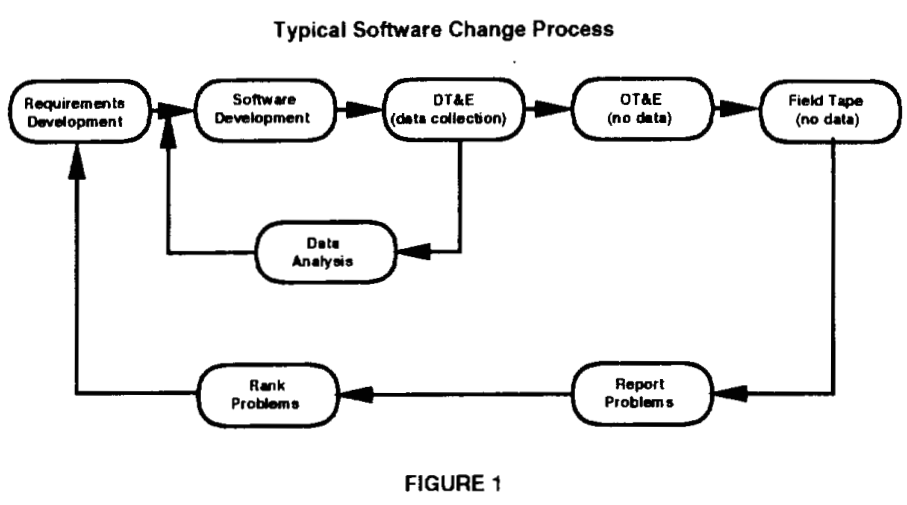
facts. programs, duplication of exact operational profiles is usually not possible. Because of the numbers of aircraft involved in tactical scenarios, more often than not, it is too expensive to create test scenarios which duplicate them.

\subsection{Current Capability Assessment}

A review of the current technology available from industry, the technology trends, and the current technologies and methodologies currently being used in the Air Force today revealed several

First, most of the technology used today for data collection is targeted to only test configurations. This emphasis, while excellent from the test only environment, was not generally adaptable to fleet aircraft of the same type. The current data collection systems require specialized compartments and, in some cases, extensive avionics modifications.

Second, the size and weight of these systems would prohibit extensive use in tactical aircraft. The inclusion of these systems in tactical aircraft would require, in some cases, the trade-off of stores or fuel which is an

prototype evaluation. This was due in part to the activities of Operations Desert Shield and Desert Storm. While it is generally accepted that test aircraft carry sufficient instrumentation to monitor and collect avionics data, this is not the case with the tactical aircraft. This presents a problem when performance anomalies occur during actual operational engagements and not under controlled test environments.

Collecting data in a tactical environment will not only facilitate turnaround of emergency OFP changes, but will also improve the overall quality of peacetime software changes. If operational aircraft were instrumented, avionics programmers would have a much better idea of how the radar responds to real-world situations. In flight test unacceptable exchange in a combat environment.

Finally, many of these systems are peculiar to a specific aircraft or avionics system. This indicates that adaptation of a given collection system to another system would be costly and strongly predicated on the similarity of the original system to the new system.

\subsection{Advantages}

Duplication of tactical missions in flight test is not cost effective. As a result tactical data acquired with operational instrumentation is very valuable because it would provide an actual recreation of the mission scenario. The location, speed, aircraft altitude, and more of both the F- 
15 and its targets will be preserved. Operationally collected data can prove invaluable to both pilots and programmers. Advantages include reduced long-term flight test costs, improved hardware fault isolation, increased probability of kill and survivability, quicker turnaround of responses to field anomalies, and a new twist on intelligence information.

Greater gains can be anticipated in the area of electronic combat. The parameters of electronic countermeasures (ECM) used by the enemy can be re-built based on the collected data. This would allow for an effective electronic countercountermeasure (ECCM) algorithm to be developed. This low-cost data should supplement the flight test data and eventually reduce the amount of flight testing required to generate ECCM algorithms.

Avionics hardware fault isolation can be greatly supplemented by the collection of communications bus data. Often line replaceable units (LRUs) fail built-in-test (BIT) on the aircraft, then pass the fault isolation test in the avionics intermediate shop (AIS). The additional data will be greatly beneficial to the engineers responsible for determining the source of this problem, that is, the aircraft, LRU, BIT software or AIS test station software.

\subsection{Impacts for Deployment}

After the flightworthy operational instrumentation prototype system has been developed and proven, the system will need to be integrated into the operational fleet. This can be accomplished through various methods.

One method may be to procure a small quantity of systems, e.g. 10 , to retrofit into an Operational Test and Evaluation (OT\&E) wing for limited implementation to further prove the system's worthiness. This method would not provide in-theatre data but would test the concept by supplying data from sources not usually seen by the avionics integrated support facilities (AISFs).
Another method may be to procure a quantity of units similar to the previous method and integrate them into a single tactical fighter wing. This method would test the true value of the system through the assessment of the field-collected data. The advantage of this method over the previous one is the data being collected. The intheatre collected data may provide valuable insight into enemy threats and their effect on our weapons systems.

While this does in no way cover the spectrum of implementation methods, it does provide an opportunity to technically and financially assess the worthiness of operational avionics instrumentation.

\subsection{An Operational Avionics Instrumentation Effort, DICE}

The need for a low cost instrumentation system which can be hosted on-board tactical aircraft to collect and record avionics data has resulted in the development of the Data Integration and Collection Environment (DICE) System currently in development by TRW Inc. under contract to Wright Laboratory, Wright-Patterson AFB OH.

The F-15 APG-63 Fire Control radar system was chosen as the testbed for the prototype. This system was selected because of its relative complexity and tactical presence in today's air combat arena.

Wright Laboratory's desire to provide an open architecture system which is affordable, expandable, adaptable, reconfigurable and maintainable led to the formulation of the basic goals and requirements for a data collection system for tactical aircraft. The basic requirements for this system are:

- Low cost (production \& life-cycle support)

- Light weight 
- Non-intrusive data collection

- High data storage capacity in digital format

- Maximized use of commercial off-the-shelf (COTS) equipment

- Adaptable to multiple platforms

- Fit into available aircraft space with minimal aircraft modifications

- Minimal logistic support for the system. methodology can be employed for other systems thus providing a quasi-standard interface. Figure 2 depicts the DICE design.

The DICE system uses state-of-the-art field reprogrammable technology to define the communication interfaces for serial and parallel data. This feature is the key to the logistics support question. The ability to modify the hardware interface configuration through software means that the DICE system can be used to instrument different systems with the same hardware and be configured via software. Additionally, this feature allows the target system to grow without requiring a new instrumentation set.

The question

of cost

effective

logistical

support of

the system is

a prime

concern for

the

prototyping

effort. The

DICE design

extensively

incorporates

current

commercial

technology.

This ensures

that as commercial technology improves, the military's technology keeps pace in a very cost effective manner.

\subsection{The DICE Design}

The prototype DICE system uses the Comptek Instrumentation Interface Unit (IIU) as a source of it's data. This approach, a two-card set added to the APG-63 avionics suite, provides an interface for collecting sufficient radar data to assist an analyst in finding and resolving anomalies at the digital level. A similar
For processing power, the current

DICE system uses a $\quad 68030$ processor in a VME backplane. This configuration allows substantial flexibility for peripheral and

processor growth and expansion. The use of a commercial standard for the processor architecture provides a stable base for keeping abreast of the direction industry takes, therefore keeping the military's technology edge sharp.

The DICE system collects MIL-STD-1553B bus communications data using a commercial bus monitor which is VME compatible. The DICE system also uses a built-in time word generator synchronized to the aircraft mission time for time-tagging the collected data (see Figure 3). 
The DICE system

also uses a commercial 8

millimeter

cartridge magnetic

tape drive for data

storage. This

allows for

compact data

storage via a

commercial

standard system.

This system can

store $5 \mathrm{~GB}$ (166 minutes) worth of filtered data at $500 \mathrm{~KB}$ sustained data rate. The system utilizes Small Computer Serial Interface (SCSI), an industry standard, for data transmission to the recorder providing flexibility to host other recording media (see Figure 4). To augment the storage of data, the DICE system uses software to intelligently filter data based on preselected criteria. The real-time software, written in Ada, allows an analyst to define what events determine the criteria for collecting data. This reduces the amount of non-essential data recorded and aids the analysts by recording only data of interest.

\section{An}

instrumentation system is not very useful if the collected data cannot be replayed in a cohesive manner for analysis. The DICE system incorporates a design capable of recording the avionics data and

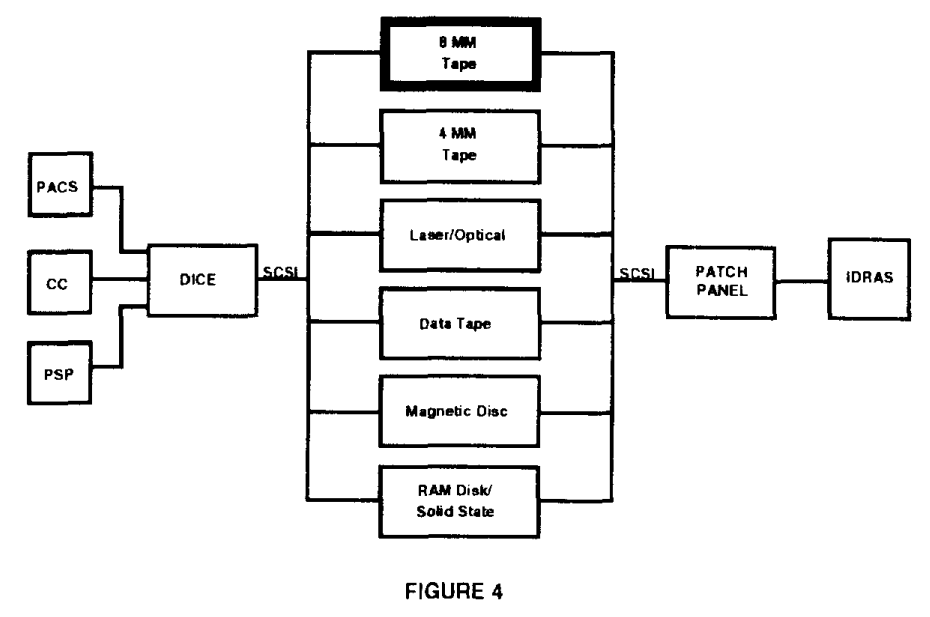

replaying it in a laboratory for analysis. This capability allows the real-time recorded data to be played back to an analysis system which is configured to receive the actual avionics bus data, or the data tape could be used to play back to another analysis tool or simulator.

\subsection{Benefits}

As is evident from the discussion of the DICE design, this system offers a highly versatile and applicable system to the area of operational avionics instrumentation. The use of the DICE system in the resolution of problems and life-cycle support are depicted in Figure 5. This figure represents a closed loop approach to OFP support and rapid turnaround of ECS software. An additional benefit not associated with the RT of OFPs but equally useful, is the collected data may be used for playback in simulation training for pilots.

\subsection{Adaptability}

The use of the reprogrammable hardware interface will provide a standard data collection system easily adaptable across services on the full spectrum of aircraft in the DoD inventory.

This includes fixed wing and rotary wing. The basic DICE concept can be adapted to other systems which contain ECS connected to peripherals via communication interfaces and their corresponding operational software. 


\subsection{Standardization}

The DICE system's adaptability to multiple platforms also provides the opportunity to allow for a standardization in the area of avionics test and analysis systems. The DICE open-ended architecture also allows for various test and data reduction and

analysis

systems to

be utilized

with a

common

collection

media which

could reduce

the

specialized

resources

currently

required

within

AISFs.

\subsection{Dual Use of the Technology}

The use of operational instrumentation is not limited to avionics systems. The need for data to analyze system performance, anomalies, processes and more is applicable to most all systems processing data. As most all systems become less hardware oriented and more software intensive, instrumentation becomes more valuable for maintenance and support of that system.

Specifically, instrumentation technology is applicable to commercial airliner surveillance systems, NASA satellites, life support systems, medical imaging and diagnostic equipment, videoon-demand, audio-on-demand, automobiles, and ground transportation systems which include a highway regulatory system. While some of these applications constitute dual use of instrumentation technology, others merit the concept of technology transfer.

\subsection{Conclusion}

The men and women who defend our country deserve and require the most advanced technology which can be provided. Operational avionics instrumentation will provide an excellent method to preserve and enhance our fighting capabilities.

The

beneficiaries of this technology -- hardware, software, and tactics ..- will realize a readiness posture unequaled in today's environment.

As a result, the development and implementation of operational avionics instrumentation provides a way to improve the readiness of embedded computer systems software.

\subsection{References}

Hughes Aircraft Co., "Radar Readiness Technologies", 1989.

TRW, "Radar Readiness Technologies", 1989.

SAIC, "Radar Support Environment ", 1990.

SAIC, "Advanced Avionics and Radar Support Environment", 1991.

Dawn Miner, "An Overview of the Concept of Operational Instrumentation for the Air Force", TFWGCON Proceedings, 1993.

Tod Reinhart and TRW/WRAL, "Data Integration and Collection Environment System", TFWGCON Proceedings, 1993. 